\title{
Optimal Inter-Element Spacing of FD- MIMO Planar Array in Urban Macrocell with Elevation Channel Modelling
}

\author{
Alidu Abubakari, Sabogu-S Raymond and Han-Shin Jo \\ Department of Electronic Engineering \\ Hanbat National University \\ Daejeon, Republic of Korea \\ [e-mail: aabubakari32@yahoo.com] \\ *Corresponding author: Han-Shin Jo
}

Received March 11, 2017; revised May 22, 2017; accepted June 19, 2017; published October 31, 2017

\begin{abstract}
Full Dimension multiple input multiple output (FD-MIMO) architecture employs a planar array design at the Base Station (BS) to provide high order multi-user MIMO (MU-MIMO) via simultaneous data transmission to large number of users. With FD-MIMO, the BS can also adjust the beam direction in both elevation and azimuth direction to concentrate the energy on the user of interests while minimizing the interference leakage to co-scheduled users in the same cell or users in the neighboring cells. In a typical highly populated macrocell environment, modelling the elevation angular characteristics of three-dimensional (3D) channel is critical to understanding the performance limits of the FD-MIMO system. In this paper, we study the throughput performance of FD-MIMO system with varying elevation angular spread and inter-element spacing using a 3D spatial channel model. Our results show that for a typical urban scenario, horizontal beamforming with correlated antenna spacing achieves optimal performance but by restricting the spread of elevation angles of departure, elevation beamforming achieves high array gain with wide inter-element spacing. We also realize significant gains due to spatial array processing via modelling the elevation domain and varying the inter-element spacing for both the transmitter and receiver.
\end{abstract}

Keywords: FD-MIMO, 3D spatial channel model, urban channel model, inter-element spacing, and elevation angles of departure restriction.

This work was partly supported by Institute for Information \& communications Technology Promotion(IITP) grant funded by the Korea government(MSIP) (No.B0722-16-0006,Cross Layer Design of Cryptography and Physical Layer Security for IoT Networks) and the Basic Science Research Program through the National Research Foundation of Korea funded by the Ministry of Science, ICT and Future Planning under Grant NRF2016R1C1B2011921. 


\section{Introduction}

There is an exponential rise in the demand of high data rates driven by the corresponding rise in affordable smartphones, the availability of cloud-based services and the many new connected devices on the market [1]. Research in [1] shows that with the current trend in wireless data demand, urban areas are expected to generate up to $60 \%$ of total mobile traffic. Thus there is the need to develop technologies which can guarantee improved user experience via higher network coverage. In June 2012, technologies including full dimension multiple input multiple output (FD-MIMO) and user specific beamforming was identified by the 3rd Generation Partnership Project (3GPP) as potential means of providing the required data capacity and spectral efficiency for next generation wireless networks.

FD-MIMO is an extension of massive MIMO [2] where a few hundreds of antennas are deployed at a base station (BS) to simultaneously support tens of users on the same timefrequency resource. It uses a 2-dimensional (2D) active antenna and other advanced signal processing techniques at the BS to improve the data capacity. It can achieve multi-user array gain of high order with the provision of effective data transmission to a large number of users in the network simultaneously. The work of [3] has shown that vertical deployment of antenna at the BS allows the utilization of the elevation domain and can provide up to $30 \%$ gain.

Research by [4], [5], [6] provides fundamental understanding on the benefits of FD-MIMO. It proved that using large planar array at the BS can achieve high order multiuser gain from high number of antennas, although it would limit the effective spacing between different antenna elements compared to conventional linear array. Also by varying the user density in the simulated network, 2-5 times cell average improvement is observed in [7]. The work of [7] also explored other challenges in implementing FD-MIMO including downlink precoding, channel quality indicator prediction and antenna virtualization. Ioannis et al [8], discussed the architecture of 2D antenna array and also port virtualization techniques which is used to create beams with wide coverage, necessary for broadcasting signals to all users within a sector. Most results thus far focused on beamforming techniques and 3D channel modelling.

Our previous work [9] explores the optimal antenna element arrangement which could harness the benefits of FD-MIMO by modelling only the elevation spread. We have shown that for equal spacing, square uniform planar array provides optimal performance in an urban marocell environment. Recently, field trials of the proof-of-concept FD-MIMO systems have been conducted successfully [10]. In order to study performance evaluation of FDMIMO, the problem of modelling the elevation spread in 3D channel is of prime importance. Previously the channel model considers the departure and arrival of multipath components in the azimuth angles only. So far elevation angle of 3D antenna arrays has been considered for different scenarios in 3GPP [11] however no complete channel model has been developed for realistic systems, hence more measurements have to be carried out. Also the available 2D channel models proposed in [12] and International Telecommunication Union (ITU) [13] are insufficient to study and completely evaluate the planar array performance.

In our research, we implement a complete 3D channel model by extending the azimuth channel statistics of the Spatial Channel Model (SCM) [14] to the elevation dimension. Our 3D SCM is then validated through extensive simulations to examine some bulk parameters 
that describe the characteristics of the channel over larger areas of several wavelengths. The architecture of the transmitter in FD-MIMO is very important as it dictates the potential gain of the system. So we study the effect of different transmitter and receiver antenna configuration on the performance of FD-MIMO system. By spacing the antenna elements sufficiently apart, it is possible for the signals received at these elements to undergo such a scattering in the propagation environment leading to either high or low correlation and thus resulting in high or low array gain respectively.

Our work introduces elevation angles of departure (eAoDs) restriction to perfectly model far-field scattering characteristics since BS antennas are well located above rooftops and as such there is no significant scattering around the BS. This also models the effect of NLOS dominance in typical urban environment. We then evaluated via sum-rate calculations, the best antenna configuration which could harness the high gain benefits of FD-MIMO taking the inter-element spacing and elevation angular spread into consideration. Results in this paper show that horizontal 16-by-1 antenna array with correlated antenna spacing provides very good performance for the typical urban case while 1-by-16 antenna array with wider spacing provides the best performance for urban channel with elevation spread limitation. The reverse results are obtained for the cell edge performance.

This paper is organized as follows; Section II introduces the FD-MIMO system model including the experimental set-up and the 3D channel model. Section III discusses an overview of beamforming in FD-MIMO and also the multi-user scheduling technique. Simulation environment and parameters, performance analysis of the FD-MIMO system are presented in Section IV. We present our conclusion in section V.

\section{3D Multicell Model}

\subsection{Transmission model}

The transmission technique employed in this paper follows the multi-user space-division multiple access (SDMA) similar to that used in LTE-A systems as depicted in Fig. 1. It generally operates on a two-steps procedure described as follows;

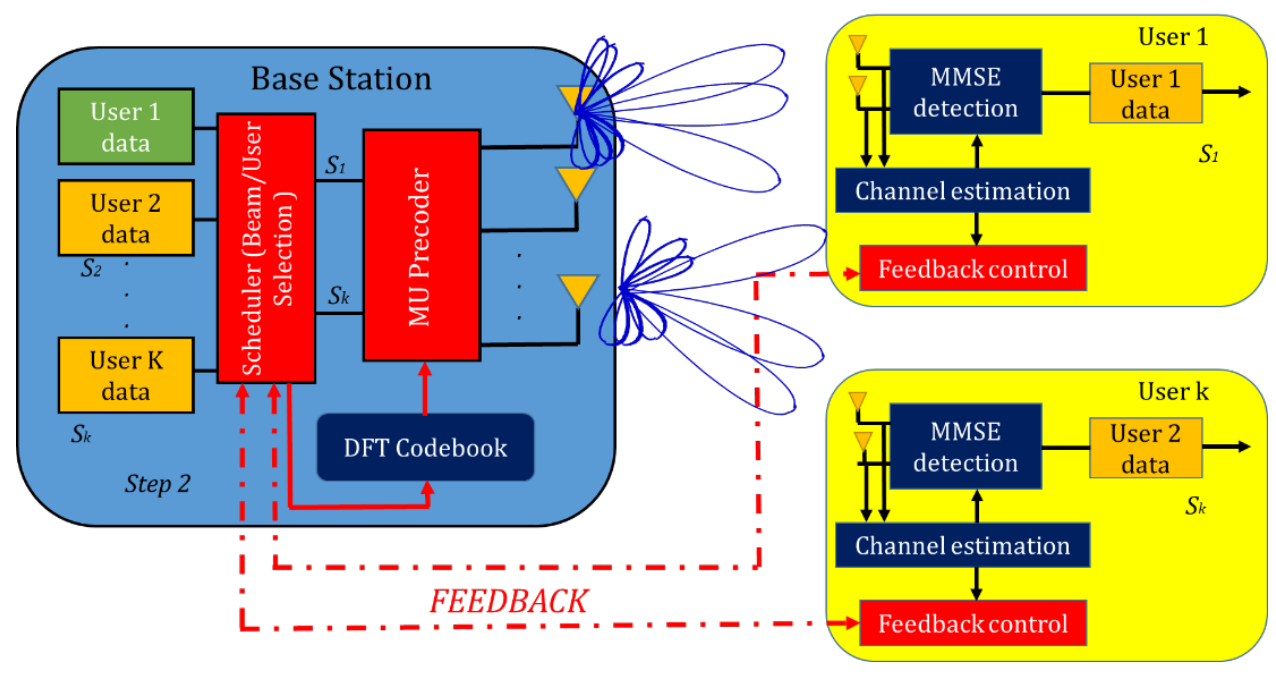

Fig. 1. Schematic illustration of the Transmission Model 
Step 1: the user in the network determines the preferred precoding matrix from the shared codebook, the post-processing signal-to-interference-ratio (SINR) and the preferred number of transmitted spatial layers periodically, then reports the rank indicator (RI), the channel quality indicator (CQI) and the precoding matrix indicator (PMI) to the serving BS by means of feedback. The PMI signals codebook index of the preferred precoding matrix and RI informs the transmitter about the number of useful spatial transmission layers for the current MIMO channel. Step 2: The base station selects the precoding matrix and the number of transmission signals based on the PMI and RI feedback for independent users. The Base station then schedules a user using a scheduling and user pairing algorithm in order to efficiently utilize the system resources. A sound methodology consisting of the cellular model, channel model and the procedure of simulation is required for the fair evaluation of proposed wireless

technologies. The simulation method for evaluating the transmission system in Fig. $\mathbf{1}$ is described by the algorithm in Fig. 2 [15]. The complete system level model in Fig. 2, defines a number of 'drops' where a mobile is placed in a sequence of different network locations. This also represents a snapshot of the fading channel.

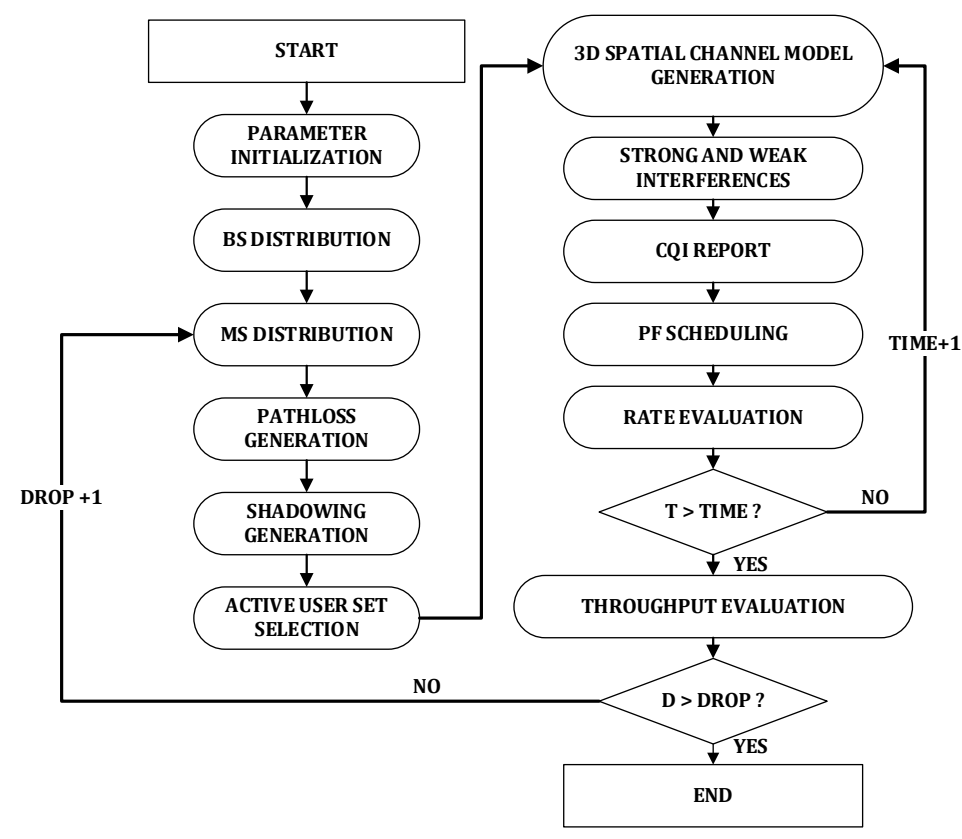

Fig. 2. System level simulation implementation

\subsection{System Model}

Our channel assumption models a typical high rise urban macro-cell scenario with line-ofsight (LOS) and Non-line-of-sight (NLOS) conditions. Users are assumed to be at street level and fixed base stations clearly above the average surrounding building heights. We consider a MIMO downlink transmission consisting of $B$ base stations and $K$ multi-antenna mobile stations in a typical homogeneous cellular network consisting of 57 cells in a two-tier deployment with a minimum inter-site distance of $500 \mathrm{~m}$. Additionally users are assumed to be randomly distributed. The $b$-th base station is equipped with $N_{T}\left(N_{V} \times N_{H}\right)$ transmit 
antennas and sends independent information to its associated MSs denoted by $k \subset\{1, \ldots, K\}$ each equipped with $N_{R}$ antennas. $N_{V}$ represents the elements in the vertical dimensioin while $N_{H}$ represents the elements in the horizontal domain.

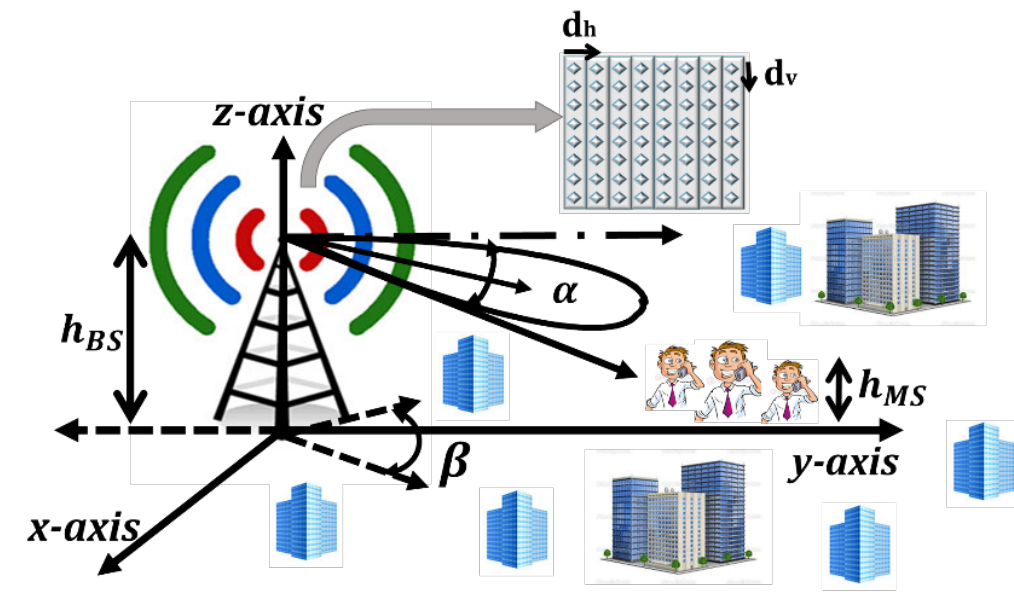

Fig. 3. Schematic illustration of the channel model

The maximum signal-strength cell association scheme is implemented such that users associate with the closest BS having the highest received power based on pathloss and shadowing [14]. We use the following notations throughout the paper: $\mathbf{A}^{\mathrm{H}}$ represents the conjugate transpose of a matrix $\mathbf{A}, \mathbf{A}^{-1}$ the inverse of a matrix $\mathbf{A}$ and $\mathbf{A}^{T}$ represents the transpose of a matrix $\mathbf{A}$. We implement an SDMA system which constructs a total of $N_{T}-1$ orthonormal beams which transmits to $N_{T}$ users $\left\{k_{p}\right\}_{p=0, \ldots, N_{T}-1}$ by means of precoding vector $\left\{\boldsymbol{w}_{p} \in \mathbb{C}^{N_{T} \times 1}\right\}_{p=0, \ldots, N_{T}-1}$. The precoded transmission signal $\mathbf{x} \in \mathbb{C}^{N_{T} \times 1}$ is given as

$$
\boldsymbol{x}=\mathbf{W s}=\sum_{\boldsymbol{p}=\mathbf{0}}^{N_{T}-1} \boldsymbol{w}_{p} s_{p}
$$

where $\mathbf{W}=\left[\mathbf{w}_{0}, \mathbf{w}_{1}, \ldots, \mathbf{w}_{N_{T}-1}\right] \in \mathbb{C}^{N_{T} \times N_{T}}$ and $\mathbf{s}=\left[\mathrm{s}_{0}, \mathrm{~s}_{1}, \ldots, \mathrm{s}_{N_{T}-1}\right]^{T}$ is the uncoded symbol vector with $E\left\{\|s\|^{2}\right\}=P_{T}$. We assume the total transmit power $P_{T}$ is equally allocated over $N_{T}$ scheduled users. The selection of $N_{T}-1$ precoding vectors are based on a beam-and-user-selection algorithm described in section 3.2. We also assume a frequency-flat block fading channel from the target BS to the $k$-th user. The received signal vector of the $k$ th user at the $b$-th base station, $y_{k ; b} \in \mathbb{C}^{N_{R} \times 1}$ is given as [16];

$$
\begin{aligned}
& y_{k ; b}=\sqrt{\frac{P_{T}}{N_{T}}} \sum_{i=1}^{B} \mathbf{H}_{k ; b, i} \sum_{j=1}^{N_{T}} \mathbf{x}_{j ; i}+\mathbf{c}_{k ; b} \\
& y_{k ; b}=\sqrt{\frac{P_{T}}{N_{T}}}\left(\begin{array}{c}
\mathbf{H}_{k ; b, b} \mathrm{w}_{k ; b} \mathrm{~s}_{k ; b}+\underbrace{\sum_{k^{\prime} \neq k} \mathbf{H}_{k ; b, b} \mathrm{w}_{k^{\prime} ; b} \mathrm{~s}_{k^{\prime} ; b}}_{\text {intracell interference }} \\
+\mathbf{c}_{k ; b}
\end{array}\right)+\underbrace{\sum_{i^{\prime} \neq b} \sum_{j=1}^{N_{T}} \sqrt{\frac{P_{T}}{N_{T}}} \mathbf{H}_{k ; b, i^{\prime}} \mathrm{w}_{j ; i^{\prime}} \mathrm{s}_{j ; i^{\prime}}}_{\text {intercell interference }}
\end{aligned}
$$


where $\boldsymbol{H}_{k ; b}$ is an $N_{R} \times N_{T}$ complex channel matrix between the $k$-th user at the $b$-th base station and $\boldsymbol{c}_{k ; b} \in N_{R} \times 1$ is a zero mean spatially white complex Gaussian receiver noise vector of covariance matrix $C_{o} \boldsymbol{I}_{N_{R}}$. Each user symbol $\left\{s_{p}\right\}_{p=0, \ldots, N_{T}-1}$ is recovered at the corresponding users via a minimum mean square error (MMSE) detection [17]. The SINR $\gamma_{k, N_{T}}$ on the $\left(N_{T}-1\right)$-th stream of the $k$-th receiver is given by

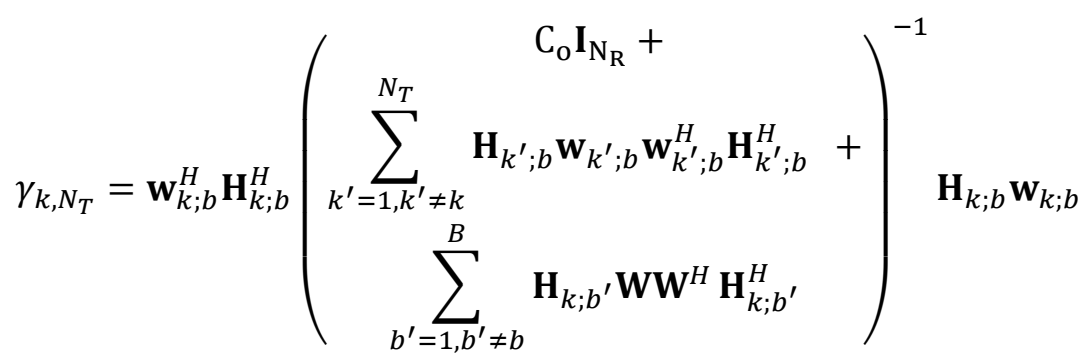

To accurately model the azimuth and elevation angle of the 3D channel [18], we used the 3D coordinate system represented in Fig. 3. Let $x_{b}, y_{b}, z_{b}$ be the coordinates of the serving 3D MIMO BS, where $x_{b}, y_{b}, z_{b}$ are the values of the $\mathrm{x}$-coordinates, $\mathrm{y}$-coordinates and $\mathrm{z}$ coordinates respectively. A similar approach i.e. $\left(x_{k ; b}, y_{k ; b}, z_{k ; b}\right)$ can be used to describe the position of the MS with respect to the BS. For convenience purposes, we define $\Delta x=x_{b}-$ $x_{k ; b}, \Delta y=y_{b}-y_{k ; b}, \Delta z=z_{b}-z_{k ; b}$ as the difference of the various coordinates between the $b$-th BS and the users. From this we can determine theoretically the distance between the BS and all the users as well as the corresponding azimuth and elevation angles as;

$$
\begin{gathered}
d_{k ; b}=\sqrt{(\Delta x)^{2}+(\Delta y)^{2}+(\Delta z)^{2}} \\
\beta=\operatorname{atan} 2(\Delta y, \Delta x) \\
\alpha=\operatorname{atan} 2\left(\Delta z, \sqrt{(\Delta x)^{2}+(\Delta y)^{2}}\right)
\end{gathered}
$$

The antenna gain which is a necessary factor to determine the large scale fading and can be determined by [19], [20];

$$
\begin{gathered}
A_{V}(\alpha)=-\min \left[12\left(\frac{\alpha-\alpha_{\text {tilt }}}{\alpha_{3 d B}}\right)^{2}, A_{m}\right] \\
A_{H}(\beta)=-\min \left[12\left(\frac{\beta}{\beta_{3 d B}}\right)^{2}, A_{m}\right]
\end{gathered}
$$

where $\alpha$ is defined as the angle between the direction of interest and the boresight of the antenna in the vertical dimension, while $\beta$ represents the same in horizontal domain. $\alpha_{3 d B}$ and $\beta_{3 d B}$ are the $3 \mathrm{~dB}$ beamwidth of the vertical and horizontal beam, respectively. $A_{m}=$ $20 \mathrm{~dB}$ is the maximum attenuation while $\alpha_{\text {tilt }}$ is the electrical tilt angle. The 3D antenna gain is combined as a sum of horizontal and vertical antenna pattern gain and is given as 


$$
G(\alpha, \beta)=G_{m}-\min \left\{-\left[A_{E, V}(\alpha)+A_{E, H}(\beta)\right], A_{m}\right\}
$$

Our simulator assumed $G_{m}=14 \mathrm{~dB}$ as the maximum directional antenna gain. Also mutual coupling among antenna elements is not considered. The macrocell distance-dependent pathloss is determined using the simplified COST231 Hata urban propagation model as reported in [14]. We adopt the log-normal shadowing model for the characterization of the shadowing effect in the 3D MIMO wireless channel.

\subsection{D Channel model}

It has been established that optimal performance characteristics of FD-MIMO to a very large extent is dependent on the channel characteristics. Favorable channel or radio propagation environment is where the propagation channel responses between transmitter and all receivers are sufficiently different. Thus, it is important to accurately model the channel behavior to be close to realistic environment measurements to achieve the full potential of MIMO. The SCM was designed for evaluating multiple antenna systems and algorithms. The model was developed within a combined 3GPP-3GPP2 ad hoc group to address the need for a precise channel model able to facilitate fair comparisons of various MIMO proposals.

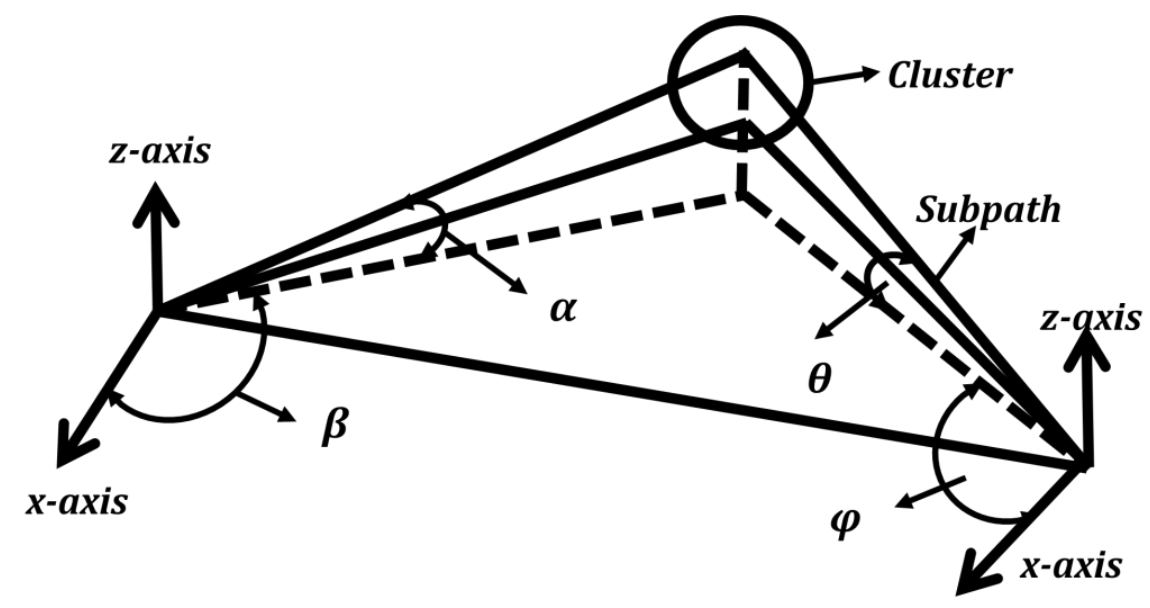

Fig. 4. 3D SCM Channel Model

The SCM specifies a set of paths between the BS and the users based on stochastic model of correlated random variables to establish the spatial, temporal and propagation characteristics for a particular channel realization. To completely understand the FD-MIMO system, it is important to model the 3D spatial channel. A simplified single-cluster illustration of the 3D SCM is shown in Fig. 4.

Currently 3GPP has developed some aspects of the 3D channel model and is in the process of finalizing the complete system. Since a detailed 3D SCM implementation is so far not available, we have constructed a working 3D SCM model by reusing the same modeling principle as the 2D SCM and by referring to the large scale fading statistics such as the elevation spread at departure, the elevation spread at arrival and the cross-correlations with other large scale fading parameters (when applicable) that are reported in [14],[21]. 


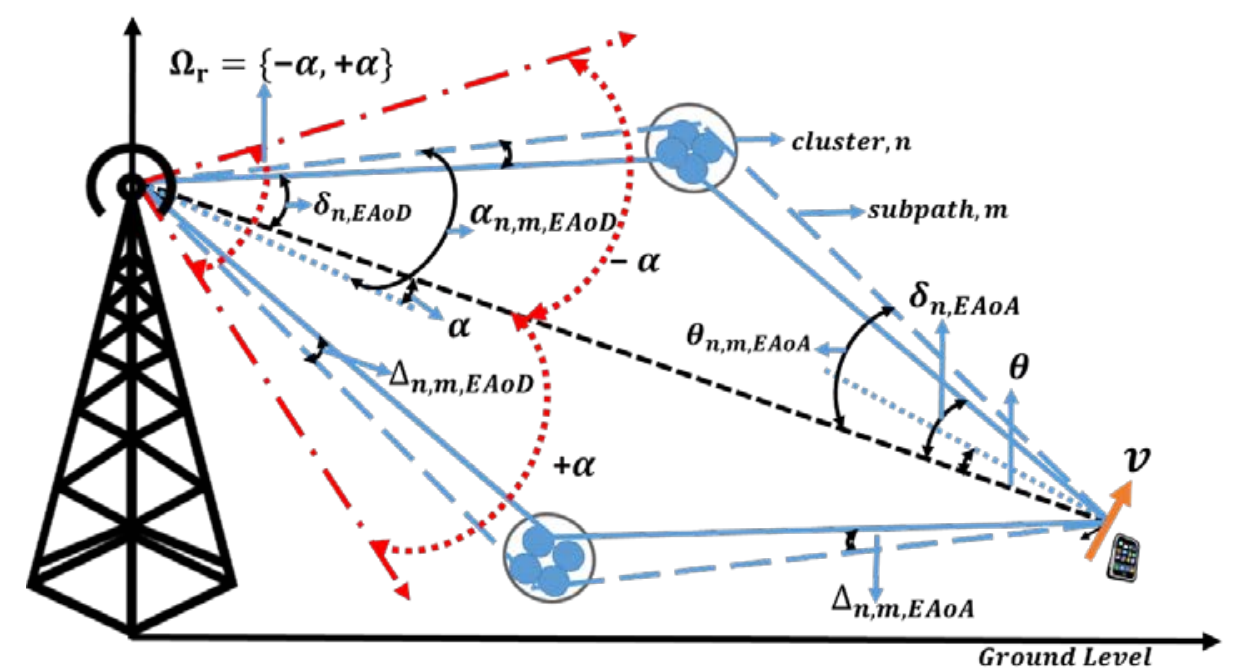

Fig. 5. Two (2)-Cluster SCM for Elevation dimension

Fig. 5, shows a two-cluster SCM channel for the elevation domain. From this figure we can define the absolute angles of departure and arrival for both the elevation and azimuth as;

$$
\begin{aligned}
& \alpha_{n, m, E A O D}=\alpha+\delta_{n, E A O D}+\Delta_{n, m, E A o D} \\
& \theta_{n, m, E A O A}=\theta+\delta_{n, E A o A}+\Delta_{n, m, E A o A} \\
& \beta_{n, m, A A O D}=\beta+\delta_{n, A A O D}+\Delta_{n, m, A A o D} \\
& \varphi_{n, m, A A O A}=\varphi+\delta_{n, A A O A}+\Delta_{n, m, A A O A}
\end{aligned}
$$

It is important to note that, the subpath angle offsets are taken from Table 5.2 in [14] and are such that;

$$
\begin{aligned}
& \Delta_{n, m, E A O D}=\Delta_{n, m, A A O D}=\Delta_{n, m, A o D} \\
& \Delta_{n, m, E A O A}=\Delta_{n, m, A A O A}=\Delta_{n, m, A o A}
\end{aligned}
$$

In our work we introduce a parameter known as the range restriction factor based on results from popular channel measurements which would model the EAoD restriction used in our work. Thus all BS EAoDs are limited to the ranges in Table 1 for each channel environment.

Table 1. Environment Elevation Range Spread

\begin{tabular}{|l|c|}
\hline \multicolumn{1}{|c|}{ Channel } & $\begin{array}{c}\text { Elevation Range Spread } \\
\Omega_{r}=\left\{\left(-\alpha^{\circ}\right) \sim\left(+\alpha^{\circ}\right)\right\}\end{array}$ \\
\hline General Urban NLOS channel & $-180^{\circ} \sim 180^{\circ}$ \\
\hline Urban NLOS channel with EAoD restriction & $-10^{\circ} \sim 10^{\circ}$ \\
\hline Urban LOS channel & $0^{\circ}$ \\
\hline
\end{tabular}

In the case of LOS urban channel; the elevation AoD restricted to single direction (in this case $0^{\circ}$ ). This is due to the fact that from channel measurements we note that $\theta>90^{\circ}$ is rarely observed, therefore we restricted the elevation AoD range to $\left[-10^{\circ}, 10^{\circ}\right]$ with respect to the LOS path between the two terminals. Also all AoDs outside the restricted angular zone is set to the LOS which in this case is $0^{0}$. Thus majority of all AoDs would be in the LOS 
direction. The fading processes for each sector and receive antenna are independent, and the doppler rate is determined by the speed of the users. We assume that the fading is equivalent for each mobile receive antenna. These assumptions are feasible and consistent with practical channel measurements recorded in literature. The overall procedure for generating the channel matrices [14] as shown in Fig. 6 consists of three basic steps:

i. Selecting environment, either general urban NLOS, urban NLOS with elevation restriction, or urban LOS

ii. Obtain the parameters to be used in simulations, associated with that environment

iii. Generate the channel coefficients based on the parameters generated.

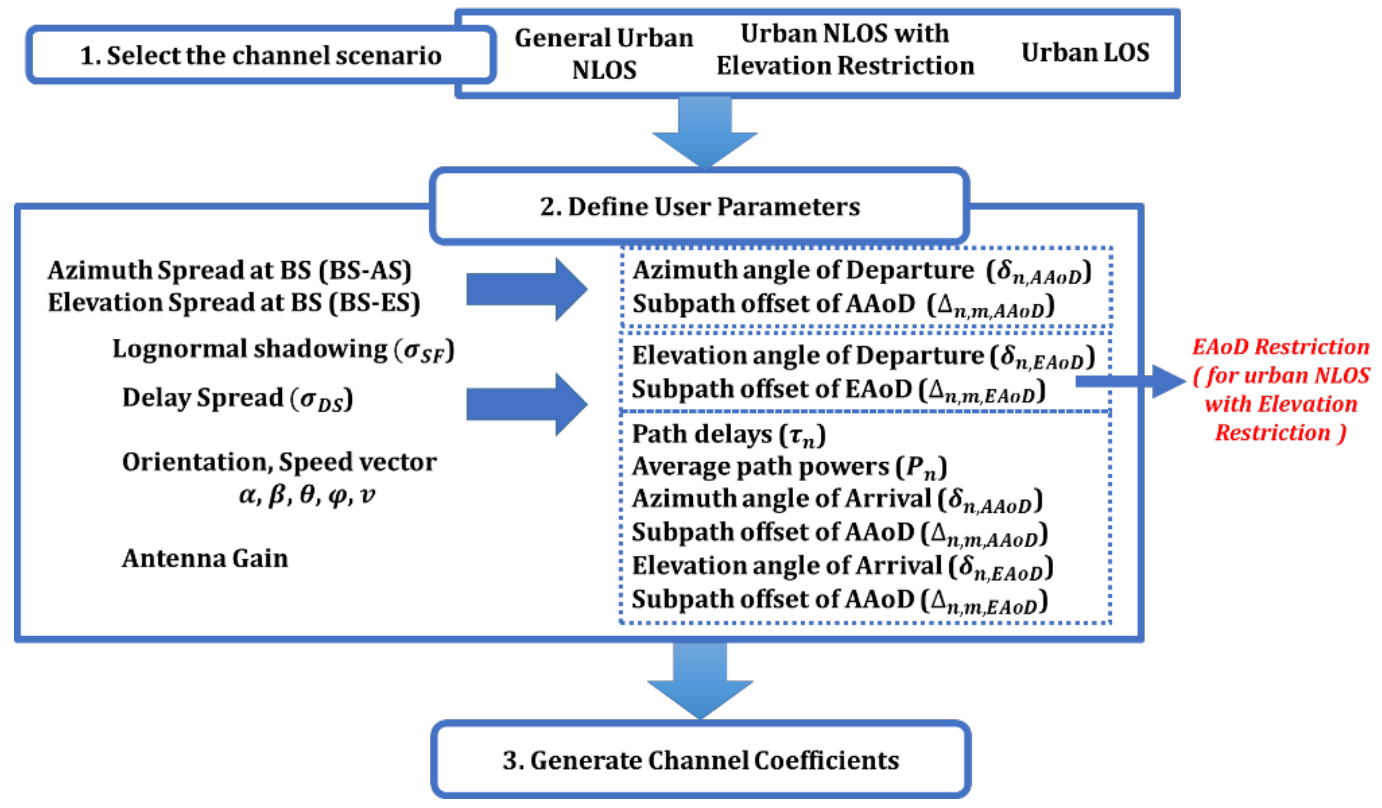

Fig. 6. Channel model overview for simulations

The channel of the $n$-th path between the $u$-th MS antenna and $s$-th BS antenna is given by

$$
h_{u, S, k}=\sqrt{\frac{p_{n} \sigma_{S F}}{M}} \sum_{m=1}^{M}\left[\begin{array}{c}
\sqrt{G_{B S}\left(\alpha_{n, m, A o D}, \beta_{n, m, A o D}\right) \times} \\
\exp \left(j\left[k d_{s} \bar{r}_{s} \overline{\phi_{n, m}}+\phi_{n, m}\right]\right) \times \\
\sqrt{G_{M S}\left(\theta_{n, m, A o A}, \varphi_{n, m, A o A}\right) \times} \\
\exp \left(j\left[k d_{u} \sin \left(\theta_{n, m, A o A}\right)\right]\right) \times \\
\exp \left(j\left[\begin{array}{l}
k\|v\| \cos \varphi_{n, m, A o A} \times \\
\cos \left(\theta_{n, m, A o A}-\theta_{v}\right) t
\end{array}\right]\right)
\end{array}\right]
$$

where

$$
\overline{\mathrm{r}_{\mathrm{s}}} \overline{\phi_{\mathrm{n}, \mathrm{m}}}=\mathrm{x}_{\mathrm{s}} \cos \beta_{n, m, A o D} \cos \alpha_{n, m, A o D}+
$$




$$
\mathrm{y}_{\mathrm{s}} \cos \beta_{n, m, A o D} \sin \alpha_{n, m, A o D}+\mathrm{z}_{\mathrm{s}} \sin \beta_{n, m, A o D}
$$

For the purposes of our simulation, we assume the elevation angular spread for typical urban macrocell case ranges from $0 \leq \theta \leq 360^{\circ}$. The notations used in the above equation are defined in Table 2 below;

Table 2. Parameter definitions

\begin{tabular}{|c|c|}
\hline Parameter & Representation \\
\hline$p_{n}$ & The power of the $n$-th path \\
\hline$\sigma_{S F}$ & $\begin{array}{l}\text { The lognormal shadow fading, applied as a bulk parameter to the } N \\
\text { paths for a given drop }\end{array}$ \\
\hline$\beta_{n, m, A A o D}$ & $\begin{array}{l}\text { Elevation domain angle of departure (EAoD) for the } m \text {-th subpath of } \\
\text { the } n \text {-th path at the BS with respect to the BS broadside }\end{array}$ \\
\hline$\varphi_{n, m, A A o A}$ & $\begin{array}{l}\text { Elevation domain angle of departure (EAoD) for the } m \text {-th subpath of } \\
\text { the } n \text {-th path at the BS with respect to the BS broadside }\end{array}$ \\
\hline$\alpha_{n, m, E A o D}$ & $\begin{array}{l}\text { Azimuth domain AoD (AAoD) for the } m \text {-th subpath of the } n \text {-th path at } \\
\text { the BS with respect to the BS broadside }\end{array}$ \\
\hline$\theta_{n, m, E A o A}$ & $\begin{array}{l}\text { Azimuth domain AoA (AAoA) for the } m \text {-th subpath of the } n \text {-th path at } \\
\text { the MS with respect to the MS broadside }\end{array}$ \\
\hline$x_{s}, y_{s}, z_{s}$ & Components of $\overline{\mathrm{r}_{\mathrm{s}}}$ to $x, y$, and $z$-axis respectively \\
\hline$\overline{\mathrm{r}_{\mathrm{S}}}$ & Location vector of Tx array element $s$ \\
\hline$d_{u}$ & $\begin{array}{l}\text { The distance in meters from MS antenna element } u \text { from the reference } \\
(u=1) \text { antenna }\end{array}$ \\
\hline$d_{s}$ & $\begin{array}{l}\text { The distance in meters from BS antenna element } s \text { from the reference } \\
(s=1) \text { antenna }\end{array}$ \\
\hline$\overline{\phi_{\mathrm{n}, \mathrm{m}}}$ & Departure angle unit vector of ray $n, m$ \\
\hline$\phi_{n, m}$ & The random phase of the $m$ th subpath of the $n$-th path \\
\hline$G_{B S}(\cdot)$ & The BS antenna gain of each array element \\
\hline$G_{M S}(\cdot)$ & The MS antenna gain of each array element \\
\hline$\theta_{v}$ & $\begin{array}{l}\text { The azimuth angle of the MS velocity vector with respect to the MS } \\
\text { broadside }\end{array}$ \\
\hline$\|v\|$ & The magnitude of the MS velocity vector \\
\hline$k$ & The wave number $\frac{2 \pi}{\lambda}$ where $\lambda$ is the carrier wavelength in meters \\
\hline
\end{tabular}

\section{3D MIMO Beamforming Technique}

The Third Generation Partnership Project (3GPP) in release 12, focused on employing improved antenna structures to improve system performance through adaptive transmission in both elevation and azimuth dimension [22]. 3D beamforming exploits the 3D MIMO channel gains in the elevation dimension as well as the azimuth dimension providing additional degree of freedom as compared to the conventional 2D antenna beamforming [23]. Control of the elevation domain would allow the independent transmission of data to specific users thereby increasing the received SINR and also minimizing the interfering power from undesired sources [24]. This concept of beamforming is where an array of antennas is used to form one or several portions within a cell with controlled antenna radiation patterns. It is a signal processing technique which involves the application of weights to adjust the phase and 
the amplitude of the transmit signals to form beam pattern towards the desired direction. This improves spectral efficiency and is especially good in cell edge user scenarios where signal power is low (i.e. low SINR).

\subsection{Kronecker Product-Based Codebook}

In order to fully exploit the performance gains of 3D MIMO employing frequency division duplexing, there is the need to design an optimal 3D codebook which takes the elevation channel state information into consideration. For highly correlated channel characteristics, the Discrete Fourier Transform (DFT)-based codebook has been proved to provide better performance in the case of both ULA and UPA. Due to its simplicity and effectiveness, it has been adopted by the 3GPP Long Term Evolution [25], [26]. The channels are highly correlated due to the fact that BS's with massive 3D antenna configuration tend to assemble large number of antennas within a limited space [27][28][29]. Thus the distances between the antennas become very small. Research in [30], showed that the correlation matrix of 3D channels can be approximated by the kronecker product of the azimuth and the elevation correlations. Using the idea of [30], [31] presented a MIMO feedback scheme based on the Kronecker product -based codebook. Basically the Kronecker product -based codebook is the product of two traditional discrete Fourier transform codebooks. The final codeword consists of the kronecker product of two oversampled DFT codewords from both the horizontal and vertical codebooks.

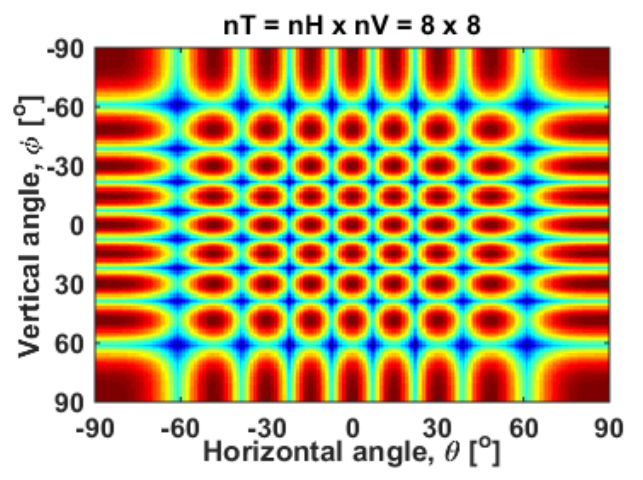

Fig. 7a. Beam pattern for 8 X8 array

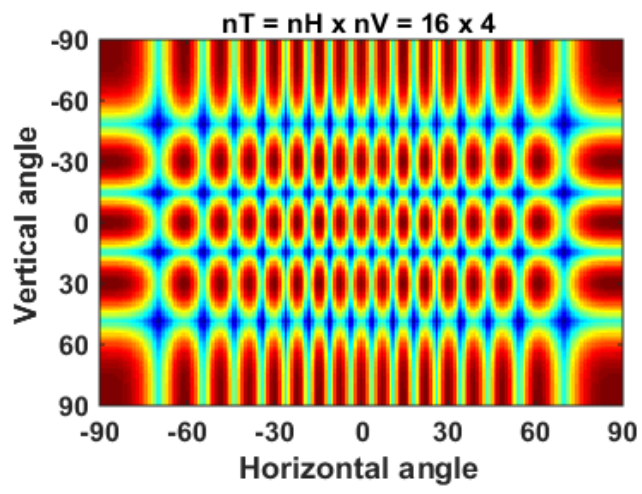

Fig. 7b. Beam pattern 16X4 array

In this design, the final codeword consists of the kronecker product of two oversampled DFT codewords from both the horizontal and vertical codebooks. Specifically $\boldsymbol{w}=$ $\left(\boldsymbol{w}_{1}, \boldsymbol{w}_{2}, \ldots, \boldsymbol{w}_{N_{v} x N_{h}}\right)$ is generated by

$$
\begin{gathered}
\boldsymbol{w}_{i, v}=\frac{1}{\sqrt{N_{v}}}\left[1, e^{\frac{j 2 \pi i}{N_{v}}}, \cdots, e^{\frac{j 2 \pi\left(N_{v}-1\right) i}{N_{v}}}\right]^{T} \\
\boldsymbol{w}_{j, h}=\frac{1}{\sqrt{N_{h}}}\left[1, e^{\frac{j 2 \pi j}{N_{h}}}, \cdots, e^{\frac{j 2 \pi\left(N_{h}-1\right) j}{N_{h}}}\right]^{T} \\
\boldsymbol{w}_{(i-1) N_{v}+j}=\boldsymbol{w}_{i, v} \otimes \boldsymbol{w}_{j, h}
\end{gathered}
$$

where $i=1,2, \ldots, N_{v} ; j=1,2, \ldots, N_{h}$; while $N_{v}$ and $N_{h}$ are the number of codewords in the horizontal and vertical codebook respectively and $\otimes$ represents the kronecker product. 


\subsection{Multi-User Scheduling with Limited Feedback}

Considering $N_{T}$ column preocoding vectors such that $\boldsymbol{W}_{p}=\left[\boldsymbol{w}_{p, 1}, \boldsymbol{w}_{p, 2}, \ldots, \boldsymbol{w}_{p, N_{T}}\right] \in$ $\mathbb{C}^{N_{T} \times N_{T}}$. Within the codebook, each selected precoding matrix corresponds to different receiver sets which directly affect the sum throughput [32][33]. Thus it is important to select an optimal precoding matrix and the corresponding user set which maximizes the data rate. This requires each user to feedback $N_{T}$ sets of $N_{T}-1$ signal to interference plus noise ratios $\left\{\gamma_{k, l}\left(\boldsymbol{W}_{p}\right)\right\}_{l=1, \ldots, N_{T}, p=0, \ldots, N_{T}-1}$. For the purposes of practicality, this SINR feedback method makes it possible to determine the optimal precoding matrix but the feedback overhead is prohibitive for implementation in an FD-MIMO system. The optimal scheme for practical implementation is the limited feedback scheme where each user only feedback $N_{T}$ SINRs $\left\{\gamma_{k, l}\left(\boldsymbol{W}_{p_{k}}\right)\right\}_{l=1 \ldots, N_{T}}$ by selecting a precoding matrix $\boldsymbol{W}_{p_{k}}$ within the shared codebook given that $p_{k} \in\left\{0, \ldots, N_{T}-1\right\}$. From our initial assumption that each user has perfect receiver CSI $\mathbf{H}_{k}$ and also share the precoder codebook; the $k$-th user selects the precoding matrix as;

$$
\boldsymbol{W}_{p_{k}}=\arg \max _{\boldsymbol{W}_{p} \in \mathbb{C}^{N_{T} \times N_{T}}} \max _{l=\left\{1, \ldots, N_{T}\right\}} \gamma_{k, l}\left(\boldsymbol{W}_{p}\right)
$$

We define $\gamma_{k, l}\left(\boldsymbol{W}_{p}\right)$ as the SINR on the $l$-th stream precoded by the $\boldsymbol{W}_{p}$ of the $k$-th user defined using the MMSE receiver in (4). Since we assume the codebook $\left\{\boldsymbol{W}_{p}\right\}_{p=0, \ldots, N_{T}-1}$ is known a priori to both the FD-MIMO BS and user, the user feeds back to the BS the index $p_{k}$ and $N_{T}$ SINRs $\left\{\gamma_{k, l}\left(\boldsymbol{W}_{p_{k}}\right)\right\}_{l=1, \ldots, N_{T}}$. All $K$ users are then grouped into $N_{T}$ groups based on their precoding matrix using the criteria

$$
\Gamma_{p}=\left\{1 \leq k \leq K \mid p_{k}=p\right\}, 1<p<N_{T}
$$

Consequently the BS tries to assign $\boldsymbol{w}_{p, l}$ within each group to a user $k_{p, l}^{*}$ with the highest SINR obtained as $\gamma_{p, l}^{*}=\max _{k \in \Gamma_{p}} \gamma_{k, l}\left(\boldsymbol{W}_{p_{k}}\right)$. The $p^{*}$-th precoding matrix $\boldsymbol{W}_{p^{*}}$ used for the transmission is selected so as to optimize the instantenoues sum throughput as follows;

$$
p^{*}=\arg \max _{p=0, \ldots, N_{T}-1} \sum_{l=1}^{N_{T}} \log _{2}\left(1+\gamma_{p, l}^{*}\right)
$$

Therefore, the final set of scheduled users specified by the set of index $\left\{k_{p^{*}, l}^{*}\right\}_{l=1, \ldots, N_{T}}$, share the $p^{*}$-th precoding matrix. This validates the information on user SINRs fed back to the BS and enables accurate prediction. The average sum throughput is given by

$$
R=\sum_{l=1}^{N_{T}} \log _{2}\left(1+\gamma_{p^{*}, l}^{*}\right)
$$

\subsection{SDMA Scheduling}

The designated target sector assigns transmission resources and opportunities to selected users by using the channel quality information. The serving BS selects total users which are less than or equal to the number of transmit antennas and have the highest scheduling priority per transmit antenna [9], [18], [34]. Thus in the $t$-th time slot, the BS selects user using the proportional fair algorithm given as

$$
k_{N_{T}}^{*}=\arg \max _{k \in\{1,2, \ldots, K\}} R_{k, N_{T}} / \bar{R}_{k}
$$

where $R_{k, N_{T}}$ is the instantaneous supported data rate for the k-th MS via $N_{T}$ transmit antenna 
while $\bar{R}_{k}$ is the average data rate of the k-th user updated periodically as

$$
\bar{R}_{k}(t+1)= \begin{cases}\left(1-\frac{1}{t_{c}}\right) \bar{R}_{k}(t)+\frac{1}{t_{c}} R_{k, N_{T}}(t) & k=k_{N_{T}}^{*} \\ \left(1-\frac{1}{t_{c}}\right) \bar{R}_{k}(t) & \text { otherwise }\end{cases}
$$

We let the $\bar{R}_{k}(t)$ is data rate for the $u$-th user scheduled in the $t$-th time slot while $t_{c}$ is the PF scheduler latency.

\section{Simulation Results and Discussions}

Using the system-level simulation techniques of the 3GPP LTE-A evaluation methodologies, the system sum-rate performance of the proposed FD-MIMO system is evaluated. Our system considered the typical urban macro deployment with varying number of transmitters and users as summarized in Table 5. The value of parameters employed in the generation of channel coefficients are also summarized in [4] and [14]. Performance of the system is measured with two metrics; cell average throughput which measures the overall system performance and the cell edge user throughput which represent the worst case UE performance. An antenna array configuration $(\mathrm{H}, \mathrm{V})$ corresponds to the horizontal and vertical elements whilst angular spread (AZ, EL) represents the angular spread in azimuth and elevation respectively. Our simulation assumes both the transmitter and receiver have full knowledge of the channel state information of the downlink channel. Also for simplicity, we assume all users are located at ground level (i.e. at $1.5 \mathrm{~m}$ height).

Table 5. System Simulation Parameters

\begin{tabular}{|l|l|}
\hline Parameter & Value \\
\hline Multi-cell layout & 19 cells each with 3 sectors in hexagon layout \\
\hline Inter-BS site minimum distance & $600 \mathrm{~m}$ (macro case) \\
\hline Tx Power & $43 \mathrm{dBm}$ \\
\hline Channel model & $3 \mathrm{D}$ SCM \\
\hline UE distribution & Uniform \\
\hline UE speed & $3 \mathrm{~km} / \mathrm{h}$ \\
\hline Scheduling delay & $0 \mathrm{~ms}$ \\
\hline Channel estimation & Ideal without error \\
\hline UE antenna number & $2,4,8$ \\
\hline Base Station antenna number & $2,4,8,16,32,64$ \\
\hline Max antenna gain & $14 \mathrm{~dB}$ \\
\hline 3 dB beamwidth & $70{ }^{\circ}(\mathrm{AZ}), 23^{\circ}(\mathrm{EL})$ \\
\hline Antenna element spacing & $0.5 \lambda, 1 \lambda, 2 \lambda, 4 \lambda$ \\
\hline
\end{tabular}




\subsection{Preliminary Results}

Preliminary system-level simulation is conducted to evaluate the performance of FD-

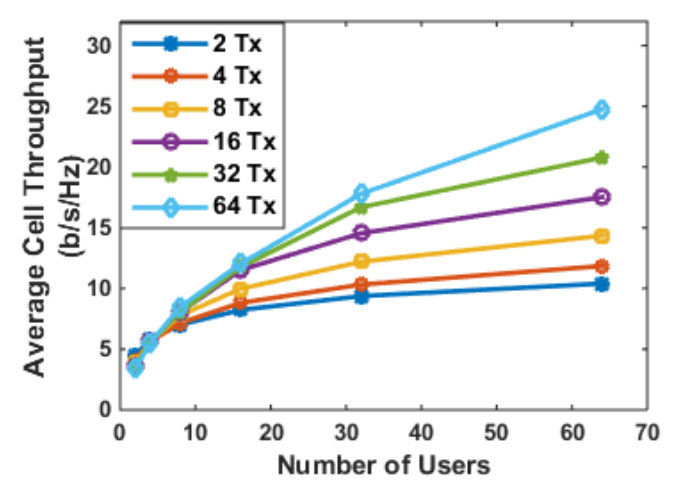

Fig. 8. Cell throughput for increasing users

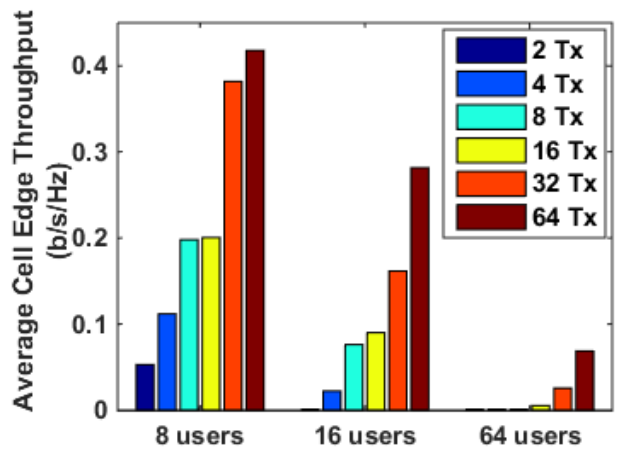

Fig. 9. Cell edge throughput with increasing users

MIMO in terms of cell average performance and cell edge with increasing number of users. Results in Fig. 8 and Fig. 9 shows the effectiveness of massive MIMO in obtaining high data rates (improving the performance of both cell edge UE and the whole system) without sacrificing bandwidth. Cell edge performance is critical in addressing the performance of users at low SINR regions. This proves the theory that large number of base station antennas can remarkably improve performance data rate. With increasing number of antennas at the base station, the propagation channel potentially provides much more spatial selectivity, from which the system performance may be greatly improved. As is expected from Fig. 9 the increase in total number of users does not improve the cell edge performance; thus there is the need to increase transmit antennas for increasing number of users.

\subsection{Throughput Performance for urban NLOS}

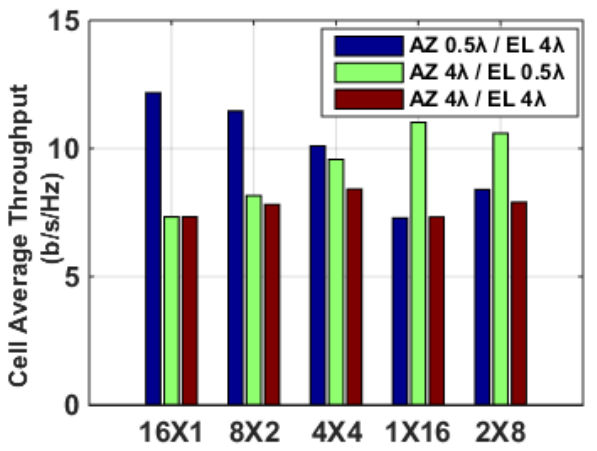

Fig. 10. Urban NLOS Sum-rate performance for $\mathrm{AZ} 8^{\circ} / \mathrm{EL} 8^{\circ}$

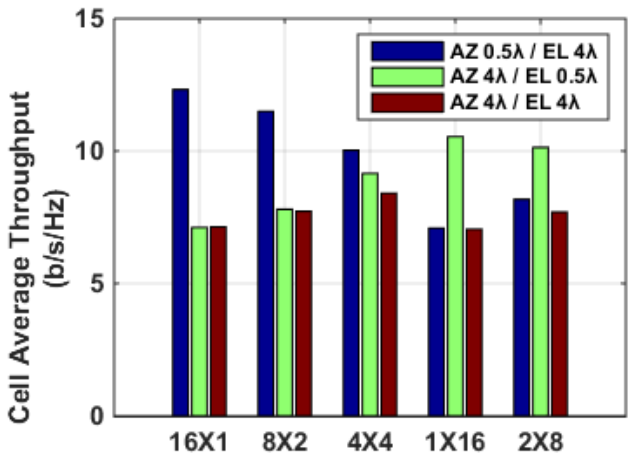

Fig. 11. Urban NLOS Sum-rate performance for $\mathrm{AZ} 8^{\circ} / \mathrm{EL} 15^{\circ}$ 


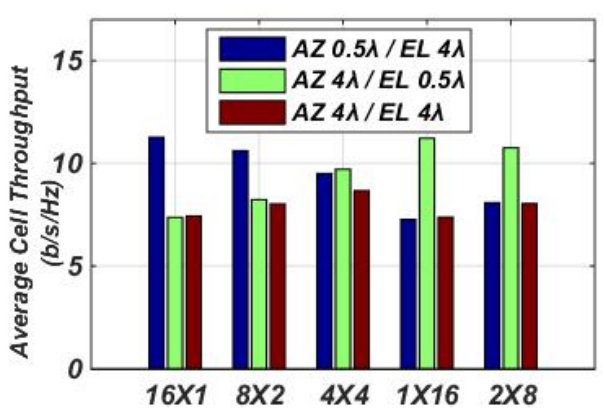

Fig. 12. Urban NLOS Sum-rate performance for $\mathrm{AZ} 15^{\circ} / \mathrm{EL} 8^{\circ}$

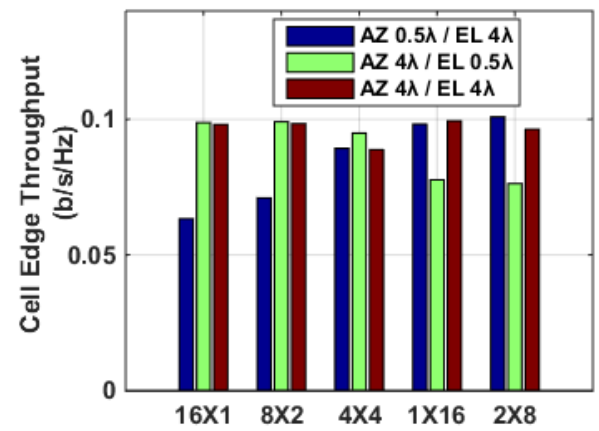

Fig. 14. Urban NLOS Cell Edge Throuput for $\mathrm{AZ} 8^{\circ} / \mathrm{EL} 15^{\circ}$

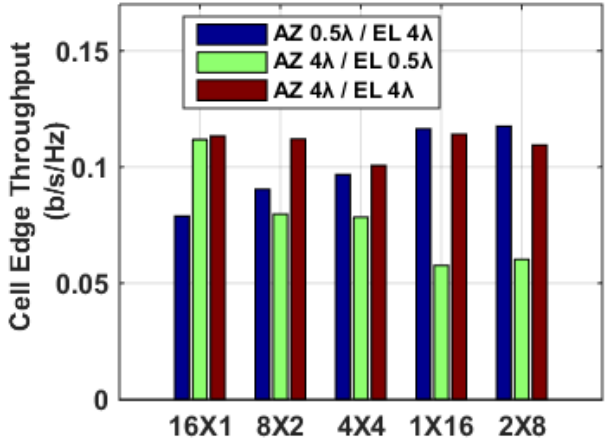

Fig. 13. Urban NLOS Cell Edge Throuput for $\mathrm{AZ} 8^{\circ} / \mathrm{EL} 8^{\circ}$

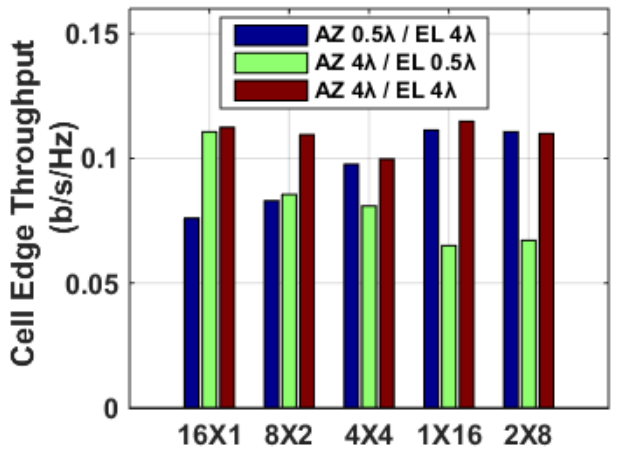

Fig. 15. Urban NLOS Cell Edge Throuput for AZ $15^{\circ} / \mathrm{EL} 8^{\circ}$

As can be observed from Fig. 10-12, the overall performance is dominated by horizontal beamforming with $0.5 \lambda / 4 \lambda$. This is because for horizontal antenna configuration with $0.5 \lambda$ inter-element spacing, multi-user interference is minimized as the beamwidth formed by the DFT precoding is very narrow. The multi-user interference is also minimized due to the fact that for the azimuth domain with sufficient user distribution it is simple to identify sufficiently spaced users improving user and beam separability.

With the small range of elevation domain, there's high probability for strong multi-user interference due to low user separability. Also high inter-beam interference can minimize the expected array gain. It can also be observed that increasing the inter-element spacing for vertical beamforming can improve performance with $1 \mathrm{x} 16$ and 2x8. As can be observed in Fig. 13-15 for the cell edge scenario, vertical antenna configuration (1x16 and 2x8) with wide inter-element spacing (4 $\lambda$ ) is optimal for all azimuth/elevation configurations. Improved performance is also observed with $8^{\circ}$ elevation spread.

\subsection{Throughput Performance for Urban NLOS with EAOD Restriction}

From Fig. 13-15, due to the elevation AoD restriction, there is increased transmission of useful signals towards the user space. The elevation domain experiences very narrow beam with high directivity. The number of uniformly spaced antenna elements employed in beamforming is directly proportional to the beamwidth for a given steering angle. Thus with the increase in the number of transmitting beams in the elevation domain, beamwidth is minimized which minimized gain loss as a result of sector-to-sector interference. 


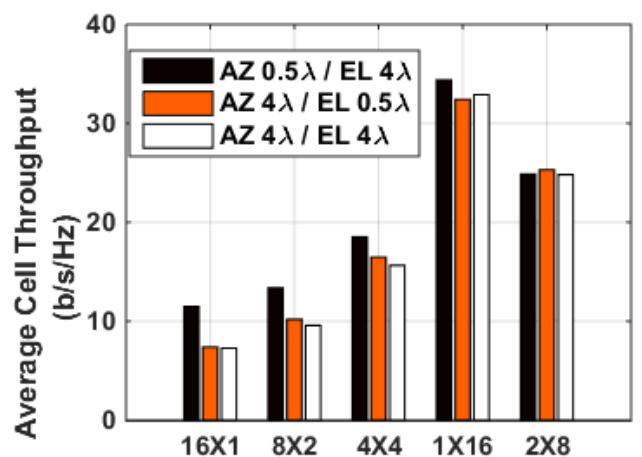

Fig. 16. Urban Restriction Sum-rate performance for AZ $8^{\circ} / \mathrm{EL} 8^{\circ}$

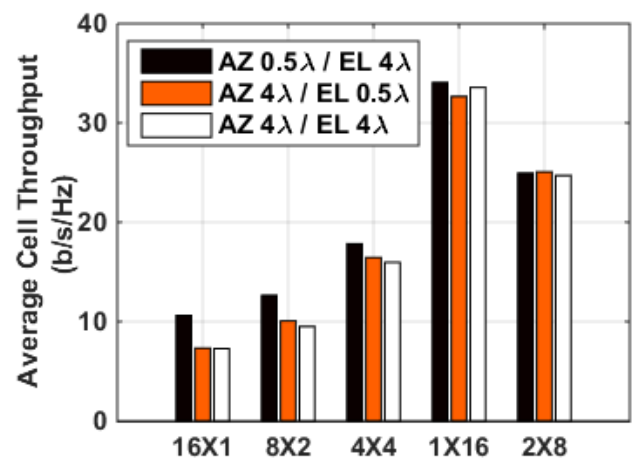

Fig. 18. Urban Restriction Sum-rate performance for AZ $15^{\circ} / \mathrm{EL} 8^{\circ}$

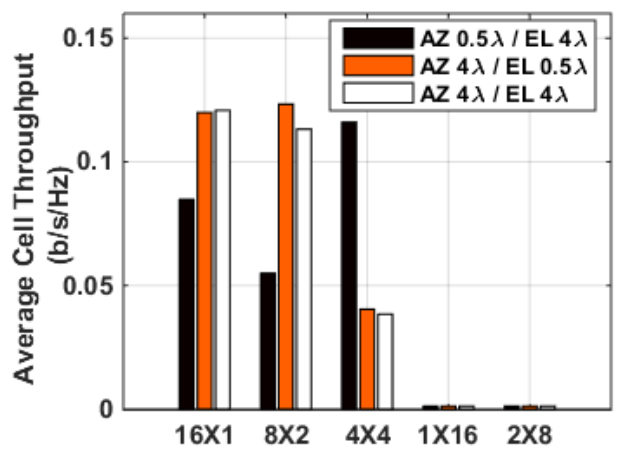

Fig. 20. Urban Restriction Cell edge throughput for $\mathrm{AZ} 8^{\circ} / \mathrm{EL} 15^{\circ}$

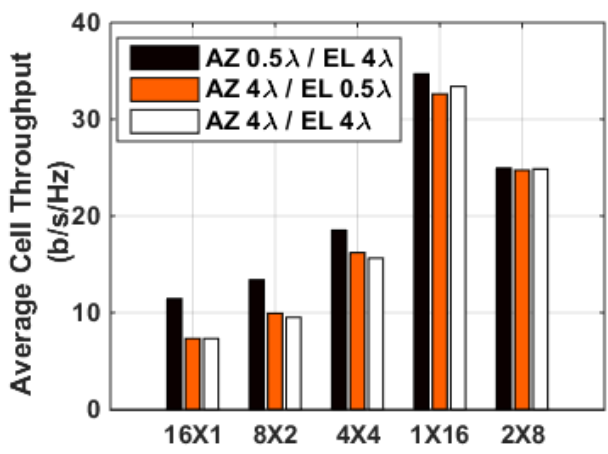

Fig. 17. Urban Restriction Sum-rate performance for AZ $8^{\circ} / \mathrm{EL} 15^{\circ}$

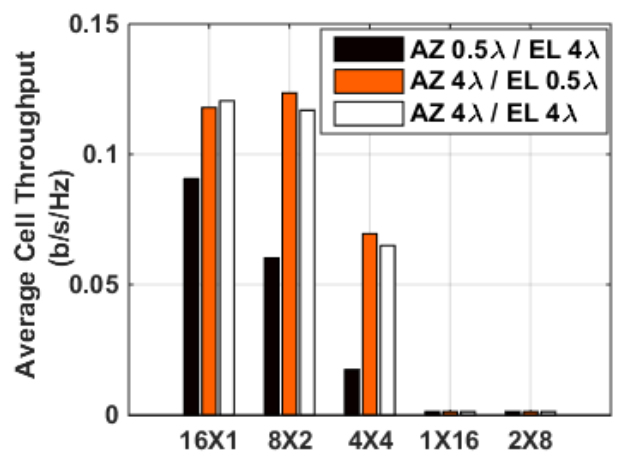

Fig. 19. Urban Restriction Cell edge throughput for $\mathrm{AZ} 8^{\circ} / \mathrm{EL} 8^{\circ}$

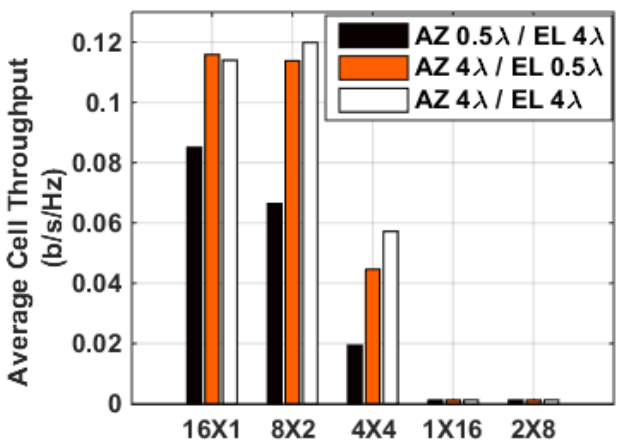

Fig. 21. Urban Restriction Cell edge throughput for $\mathrm{AZ} 15^{\circ} / \mathrm{EL} 8^{\circ}$

The high directivity prevents losses due to inter-beam overlap. However horizontal beamforming with $16 \times 1$ with $4 \lambda / 4 \lambda$ has the worst performance. The case of cell edge as observed in Fig. 19-21, horizontal beamforming $16 \times 1$ and $8 \times 2$ with $4 \lambda / 4 \lambda$ and $4 \lambda / 0.5 \lambda$ are the optimal antenna configuration for the azimuth/Elevation configuration It can also be observed that varying the inter-element spacing in the elevation domain does not adversely affect sum-rate adversely; thus beamforming performs well both under correlated and uncorrelated inter-element spacing for the case of eAoD restriction. 


\subsection{Throughput Performance for Urban LOS}

In the urban LOS scenario where elevation AoD restricted to single direction (in this case $0^{\circ}$ ), results obtained in Fig. 22-24 are similar to those obtained in the case of urban NLOS with eAoD restricted to $\left[-10^{\circ}, 10^{\circ}\right]$. Thus that vertical beamforming dominates performance for all inter-element spacing cases. This can be attributed to the elevation domain experiencing very narrow beam with high directivity as a result of the EAoD restriction. Elevation domain channel modelling through the restriction of AoDs maintains a strong spatial correlation with varying inter-element spacing.

An analysis of the results also shows that performance is large dependent on the varying inter-element spacing and the distribution of the AoDs and not necessarily the mean angular spread.

For cell edge throughput (Fig. 25-27), horizontal beamforming $16 \times 1$ and $8 \times 2$ with $4 \lambda / 4 \lambda$ and $4 \lambda / 0.5 \lambda$ respectively are the optimal antenna configuration for the azimuth/Elevation configuration as in the case of urban LOS. Vertical beamforming with $1 \times 16$ antenna configuration has the least performance due to the fact that narrow elevation beams are mostly directed towards MS's close to the cell center.

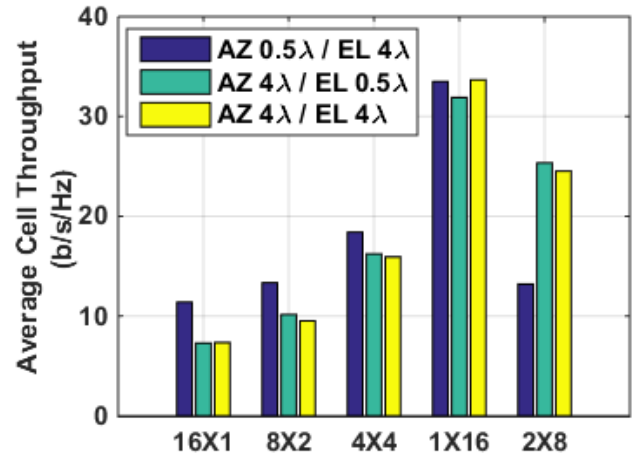

Fig. 22. Urban LOS Sum-rate performance for $\mathrm{AZ} 8^{\circ} / \mathrm{EL}^{\circ}$

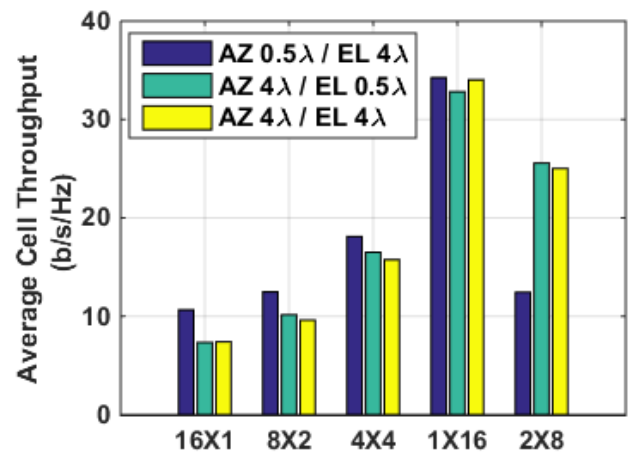

Fig. 24. Urban LOS Sum-rate performance for $\mathrm{AZ} 15^{\circ} / \mathrm{EL}^{\circ}$

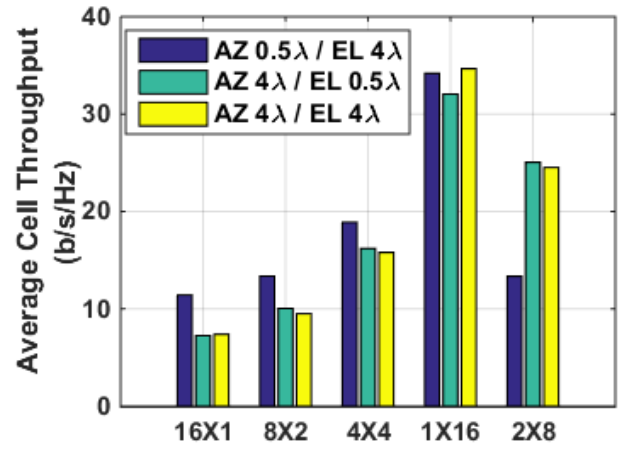

Fig. 23. Urban LOS Sum-rate performance for $\mathrm{AZ} 8^{\circ} / \mathrm{EL} 15^{\circ}$

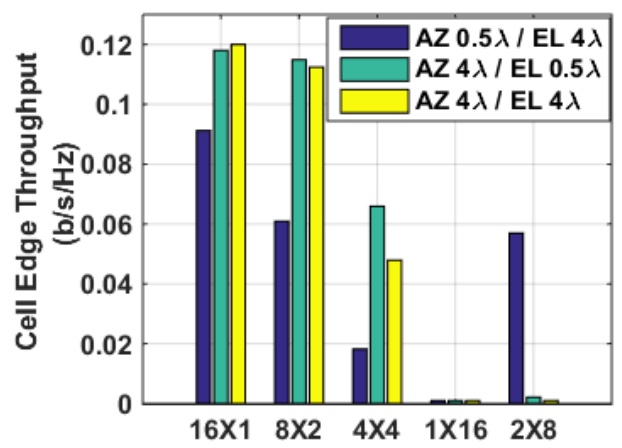

Fig. 25. Urban LOS Cell edge throughput for $\mathrm{AZ} 8^{\circ} / \mathrm{EL} 8^{\circ}$ 


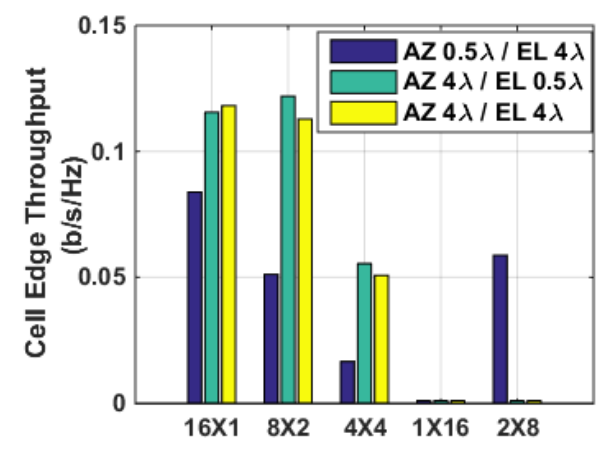

Fig. 26. Urban LOS Cell edge throughput for $\mathrm{AZ} 8^{\circ} / \mathrm{EL} 15^{\circ}$

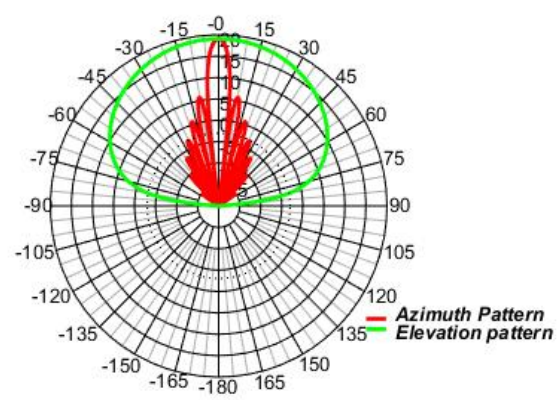

Fig. 28a. Optimal planar array pattern for urban without EAoD restriction

(16x1 with $0.5 \lambda / 4 \lambda)$

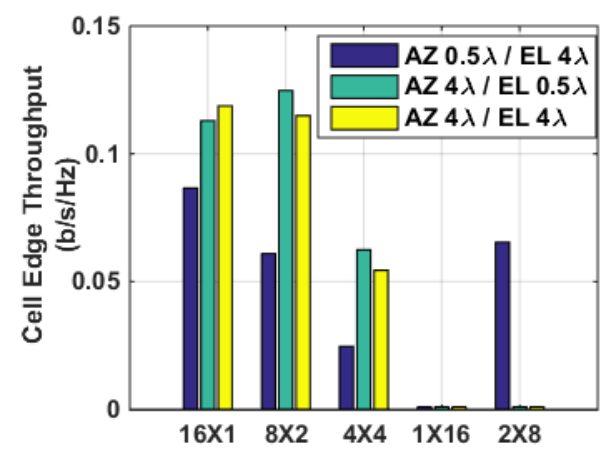

Fig. 27. Urban LOS Cell edge throughput for $\mathrm{AZ} 15^{\circ} / \mathrm{EL} 8^{\circ}$

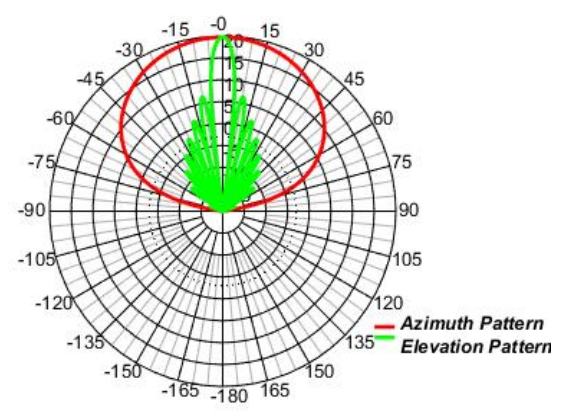

Fig. 28b. Optimal planar array pattern for urban with EAoD restriction (1x16 with $0.5 \lambda / 4 \lambda)$

\subsection{Throughput Performance with varying receiver inter-element spacing}

We present analysis of the performance of our FD-MIMO system with varying transmit and receiver antennas. The various transmit/receive architecture presented in the results are shown in Table 5 below. From Fig. 29 with the elevation angle of departure restriction, 2x4 correlated transmit antennas and 4 correlated receive antennas outperforms other configurations. For the case of no elevation restriction in Fig. 30, optimal configuration is observed for increased receiver antenna i.e. 2 X2 $(0.5 \lambda) / 8(0.5 \lambda)$.

Comparatively, improved throughput performance is obtained with increased MS antennas for typical urban NLOS case. Due to MS antenna design restriction, increasing the number of BS antenna elements with correlated inter-element spacing is sure to provide optimal performance.

Table 5. Results for Varying Tx/Rx Structure

\begin{tabular}{|l|l||l|l|}
\hline Case & Tx / Rx Structure & Case & Tx / Rx Structure \\
\hline A & $4 \mathrm{X} 4(0.5 \lambda) / 2(0.5 \lambda)$ & G & $2 \mathrm{X} 2(0.5 \lambda) / 8(0.5 \lambda)$ \\
\hline $\mathbf{B}$ & $4 \mathrm{X} 4(0.5 \lambda) / 2(2.0 \lambda)$ & $\mathbf{H}$ & $2 \mathrm{X} 2(2.0 \lambda) / 8(0.5 \lambda)$ \\
\hline $\mathbf{C}$ & $4 \mathrm{X} 4(1.0 \lambda) / 2(4.0 \lambda)$ & $\mathbf{I}$ & $2 \mathrm{X} 2(4.0 \lambda) / 8(0.5 \lambda)$ \\
\hline $\mathbf{D}$ & $2 \mathrm{X} 4(0.5 \lambda) / 4(0.5 \lambda)$ & $\mathbf{J}$ & $4 \mathrm{X} 2(0.5 \lambda) / 4(0.5 \lambda)$ \\
\hline $\mathbf{E}$ & $2 \mathrm{X} 4(0.5 \lambda) / 4(1.0 \lambda)$ & $\mathbf{K}$ & $4 \mathrm{X} 2(0.5 \lambda) / 4(1.0 \lambda)$ \\
\hline $\mathbf{F}$ & $2 \mathrm{X} 4(1.0 \lambda) / 4(2.0 \lambda)$ & $\mathbf{L}$ & $4 \mathrm{X} 2(1.0 \lambda) / 4(2.0 \lambda)$ \\
\hline
\end{tabular}




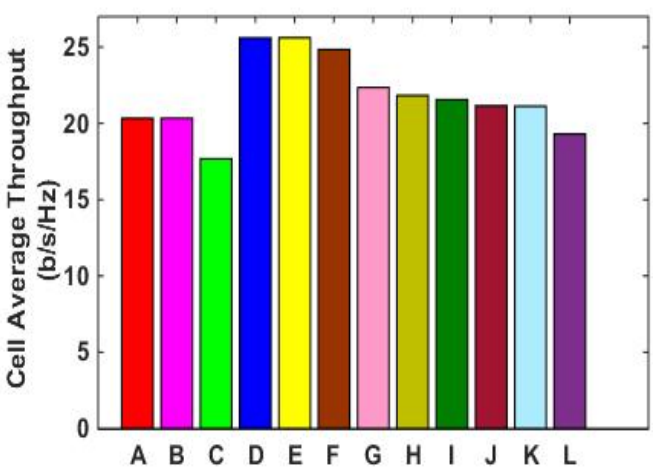

Fig. 29. Varying transmit/receiver architecture for the case of Urban Restriction

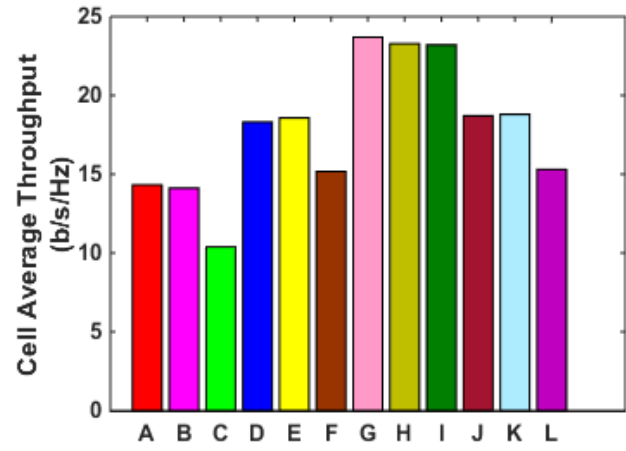

Fig. 30. Varying transmit/receiver architecture for the case of Urban without Restriction

\section{CONCLUSION}

In this paper we analyse the sum-rate performance of practical planar array configuration for FD-MIMO in urban 3D environment using system level simulations. Typically MUMIMO with 3D beamforming can work well in both correlated and uncorrelated antenna structures but for the case of urban macrocell channel with users located at street level, cell average performance is optimal with horizontal linear array with small inter-element spacing $(0.5 \lambda)$. By assuming zero mutual coupling effect between antenna elements, half-wavelength spacing would be the ideal choice to maintain high spatial correlation without greatly increasing the physical dimensions of the array. By restricting the elevation angles of departure, higher array gain is obtained via wide elevation inter-element spacing. The performance trend for cell edge is opposite as typical urban supports high cell edge throughput with vertical antenna beamforming whilst for the case or restriction, horizontal antenna beamforming is optimal. In designing the transmit grid of beams for FD-MIMO, it is important to design the antenna array and choose optimal configurations including interelement spacing for different angular spread scenarios since performance is sensitive to interference. Results in [5],[6],[7],[8]and [35] shows that for small angular spread value (typically a fixed value), the azimuth beaforming performs better than the elevation. It was proposed in [4-8] that an increase in the angular spread and inter-element spacing in the elevation domain would increase the elevation beamforming gain. Our work tested varying angular spread and inter-element spacing for both horizontal and vertical domain. We observed a similar pattern where the increase in the angular spread and elevation interelement spacing increasing the beamforming gain. Table 6 presents a summary of the results discussed in this paper. 
Table 6. Summary of Results

\begin{tabular}{|c|c|c|c|c|c|c|}
\hline \multirow{3}{*}{$\begin{array}{c}\text { Channel } \\
\text { Model }\end{array}$} & \multicolumn{3}{|c|}{ Cell Average Throughput (b/s/Hz) } & \multicolumn{3}{|c|}{ Cell Edge Throughput (b/s/Hz) } \\
\hline & \multicolumn{3}{|c|}{ Angular Spread Variations (AZ / EL) } & \multicolumn{3}{|c|}{ Angular Spread Variations (AZ / EL) } \\
\hline & $8^{\circ} / 8^{\circ}$ & $8^{\circ} / 15^{\circ}$ & $15^{\circ} / 8^{\circ}$ & $8^{\circ} / 8^{\circ}$ & $8^{\circ} / 15^{\circ}$ & $15^{\circ} / 8^{\circ}$ \\
\hline $\begin{array}{l}\text { Urban } \\
\text { NLOS }\end{array}$ & $\begin{array}{c}16 X 1 \\
(0.5 \lambda / 4 \lambda)\end{array}$ & $\begin{array}{c}16 \times 1 \\
(0.5 \lambda / 4 \lambda)\end{array}$ & $\begin{array}{c}16 X 1 \\
(0.5 \lambda / 4 \lambda)\end{array}$ & $\begin{array}{c}2 \mathrm{X} 8 \\
(0.5 \lambda / 4 \lambda)\end{array}$ & $\begin{array}{c}2 \mathrm{X} 8 \\
(0.5 \lambda / 4 \lambda)\end{array}$ & $\begin{array}{c}1 \mathrm{X} 16 \\
(4 \lambda / 4 \lambda)\end{array}$ \\
\hline $\begin{array}{c}\text { Urban } \\
\text { NLOS with } \\
\text { Restriction }\end{array}$ & $\begin{array}{c}1 \times 16 \\
(0.5 \lambda / 4 \lambda)\end{array}$ & $\begin{array}{c}1 \mathrm{X} 16 \\
(0.5 \lambda / 4 \lambda)\end{array}$ & $\begin{array}{c}1 \mathrm{X} 16 \\
(0.5 \lambda / 4 \lambda)\end{array}$ & $\begin{array}{c}8 X 2 \\
(4 \lambda / 0.5 \lambda)\end{array}$ & $\begin{array}{c}8 X 2 \\
(4 \lambda / 0.5 \lambda)\end{array}$ & $\begin{array}{c}8 X 2 \\
(4 \lambda / 4 \lambda)\end{array}$ \\
\hline $\begin{array}{l}\text { Urban } \\
\text { LOS }\end{array}$ & $\begin{array}{c}1 \mathrm{X} 16 \\
(4 \lambda / 4 \lambda)\end{array}$ & $\begin{array}{c}1 \mathrm{X} 16 \\
(4 \lambda / 4 \lambda)\end{array}$ & $\begin{array}{c}1 \mathrm{X} 16 \\
(4 \lambda / 4 \lambda)\end{array}$ & $\begin{array}{c}16 X 1 \\
(4 \lambda / 4 \lambda)\end{array}$ & $\begin{array}{c}8 X 2 \\
(4 \lambda / 0.5 \lambda)\end{array}$ & $\begin{array}{c}8 X 2 \\
(4 \lambda / 0.5 \lambda)\end{array}$ \\
\hline
\end{tabular}

\section{References}

[1] Ericsson Traffic and Market Report On the Pulse of the Networked Society, June 2012. Article (CrossRef Link)

[2] T. Marzetta, "Noncooperative cellular wireless with unlimited numbers of base station antennas,” IEEE Trans. Wireless Commun., vol. 9, no. 11, pp. 3590-3600, Nov. 2010. Article (CrossRef Link)

[3] L. Voukko, V.-M. Kolmonen, J. Kivinen, and P. Vailnikainen, "Results from $5.3 \mathrm{GHz}$ MIMO Measurement Campaign,” presented at COST 273 TD (04) 193, 2004.

Article (CrossRef Link)

[4] Y. H. Nam et al., "Full-dimension MIMO (FD-MIMO) for next generation cellular technology," IEEE Communications Magazine, vol. 51, no. 6, pp. 172-179, June 2013. Article (CrossRef Link)

[5] B. L. Ng et al., "Fulfilling the promise of massive MIMO with 2D active antenna array," IEEE Globecom Workshops (GC Wkshps), pp. 691-696, 2012. Article (CrossRef Link)

[6] Y. Kim et al., "Full dimension mimo (FD-MIMO): the next evolution of MIMO in LTE systems," IEEE Wireless Communications, vol. 21, no. 2, pp. 26-33, April 2014. Article (CrossRef Link)

[7] Y. Li et al., "Implementation of full-dimensional MIMO (FD-MIMO) in LTE," in Proc. of Asilomar Conference on Signals, Systems and Computers, pp. 998-1003, 2013. Article (CrossRef Link)

[8] I. Tzanidis, Y. Li, G. Xu, J-Y. Seol, and J. Zhang, "2D Active Antenna Array Design for FDMIMO System and Antenna Virtualization Techniques," International Journal of Antennas and Propagation, vol. 2015, Article ID 873530, 9 pages, 2015. Article (CrossRef Link)

[9] A. Abubakari, S. S. Raymond and H. S. Jo, "Full dimension MIMO Antenna Configuration for Optimal Performance," in Proc. of 6th Intl. Conference on Information and Communication Technology Convergence (ICTC), pp. 1025-1030, 2015. Article (CrossRef Link)

[10] W. Zhang, J. Xiang, Y.R. Li, Y. Wang, Y. Chen, P. Geng and Z. Lu, "Field Trial and Future Enhancements for TDD Massive MIMO Networks,” in Proc. of 26th Intl. Symp. on Personal, Indoor, and Mobile Radio Comm. (PIMRC) Workshop Advancements in Massive MIMO, pp. 1114-1118, 2015. Article (CrossRef Link) 
[11] 3GPP TR 36.873 V12.1.0 (2015-03) Article (CrossRef Link)

[12] TR 36.814, "Further advancements for E-UTRA physical layer aspects”, V 9.0.0, 3GPP. Article (CrossRef Link)

[13] ITU M.2135, "Guidelines for evaluation of radio interface technologies for IMT-Advanced," ITU-R, Dec. 2009. Article (CrossRef Link)

[14] 3GPP, TR 25.996, "Spatial channel model for multiple input multiple output (MIMO) simulations (Rel. 10),” 2011. Article (CrossRef Link)

[15] Y. Wang, J. Xu and L. Jiang, "Challenges of System-Level Simulations and Performance Evaluation for 5G Wireless Networks," IEEE Access, vol. 2, pp. 1553-1561, 2014. Article (CrossRef Link)

[16] Dorra Ben Cheikh Battikh. “Outage probability formulas for cellular networks : contributions for MIMO, CoMP and time reversal features” Telecom ParisTech, 2012. Article (CrossRef Link)

[17]F. Boccardi, H. Huang and M. Trivellato, "Multiuser eigenmode transmission for mimo broadcast channels with limited feedback," in Proc. of 2007 IEEE 8th Workshop on Signal Processing Advances in Wireless Communications, Helsinki, pp. 1-5, 2007. Article (CrossRef Link)

[18] Alidu Abubakari, Sabogu-Sumah Raymond, and Han-Shin Jo, "Optimal Planar Array Architecture for Full-Dimensional Multi-user Multiple-Input Multiple-Output with Elevation Modeling," ETRI Journal, vol. 39, no. 2, pp. 234-244, Apr. 2017. Article (CrossRef Link)

[19] “Guidelines for evaluation of radio interface technologies for IMT-advanced”, 2008, [online] Article (CrossRef Link).

[20] A. Kammoun, H. Khanfir, Z. Altman, M. Debbah, M. Kamoun, "Preliminary results on 3D channel modeling: From theory to standardization," IEEE J. Sel. Areas Commun., vol. 32, no. 6, pp. 1219-1229, June 2014. Article (CrossRef Link)

[21] G. Calcev et al., "A Wideband Spatial Channel Model for System-Wide Simulations," IEEE Transactions on Vehicular Technology, vol. 56, no. 2, pp. 389-403, March 2007. Article (CrossRef Link)

[22] Overview of 3GPP Release 12 V 0.0.9 (2013-06). Article (CrossRef Link)

[23] Y. Li, X. Ji, D. Liang, and Y. Li, “An Enhanced Beamforming Algorithm for Three Dimensional MIMO in LTE-Advanced Networks," International Conference on Wireless Communications \& Signal Processing (WCSP), pp. 24-26, Oct. 2013. Article (CrossRef Link)

[24] Y. Song, X. Yun, S. Nagata and L. Chen, "Investigation on elevation beamforming for future LTE-Advanced," IEEE International Conference on Communications Workshops (ICC), pp. 106-110, 2013. Article (CrossRef Link)

[25] J. Zhu, J. Liu, X She, L Chen "Investigation on precoding techniques in E-UTRA and proposed adaptive precoding scheme for MIMO systems," in Proc. of 14th Asia Pacific Conference on Communications (APCC), 1-5, 2008. Article (CrossRef Link)

[26] D J Love, R W Heath. "Equal gain transmission in multiple-input multiple-output wireless systems,” IEEE Transactions on Communications, Vol. 51 no. 7, pp 1102-1110, 2003. Article (CrossRef Link)

[27] S. K. Yong and J. S. Thompson, "Three-dimensional spatial fading correlation models for compact MIMO receivers," IEEE Transactions on Wireless Communications, vol. 4, no. 6, pp. 2856-2869, Nov. 2005. Article (CrossRef Link)

[28] S. Shen, M. R. McKay and R. D. Murch, "MIMO systems with mutual coupling: How many antennas to pack into fixed-length arrays?," International Symposium on Information Theory and its Applications (ISITA), pp. 531-536, 2010. Article (CrossRef Link)

[29]D. J. Love, J. Choi, P. Bidigare, “A closed-loop training approach for massive MIMO beamforming systems," in Proc. of 47th Annual Conference on Information Sciences and Systems (CISS), pp 1-5, 2013. Article (CrossRef Link)

[30]D. Ying, F W Vook, T A Thomas, D J Love, A. Ghosh "Kronecker product correlation model and limited feedback codebook design in a 3D channel model," in Proc. of 2014 IEEE International Conference on Communications (ICC), 5865-5870, 2014. 
Article (CrossRef Link)

[31] Y. Xie, S. Jin, J. Wang , Y Zhu, X. Gao, Y. Huang. “A limited feedback scheme for 3D multiuser MIMO based on Kronecker product codebook," in Proc. of 24th International Symposium on Personal Indoor and Mobile Radio Communications (PIMRC), 1130-1135, 2013. Article (CrossRef Link)

[32] H. S. Jo and C. Mun, "Transmit-nulling SDMA for coexistence with fixed wireless service”, Journal of Electromagnetic Engineering and Science, vol. 11, 2011, pp. 34-41 Article (CrossRef Link)

[33] H-S. Jo, "Spectrum Sharing SDMA with Limited Feedback: Throughput Analysis," KSII Transactions on Internet and Information Systems, vol. 6, no. 12, pp. 3237-3256, 2012. Article (CrossRef Link)

[34]Wang, H. , Meng, W. and Nguyen, T., "User Fairness Scheme with Proportional Fair Scheduling in Multi-user MIMO Limited Feedback System,” Communications and Network, 5, 113-118, 2013. Article (CrossRef Link)

[35] Younsun Kim, Hyoungju Ji, Hyojin Lee, Juho Lee, Boon Loong Ng and Jianzhong Zhang, "Evolution beyond LTE-advanced with Full Dimension MIMO," in Proc. of 2013 IEEE International Conference on Communications Workshops (ICC), Budapest, pp. 111-115, 2013. Article (CrossRef Link)
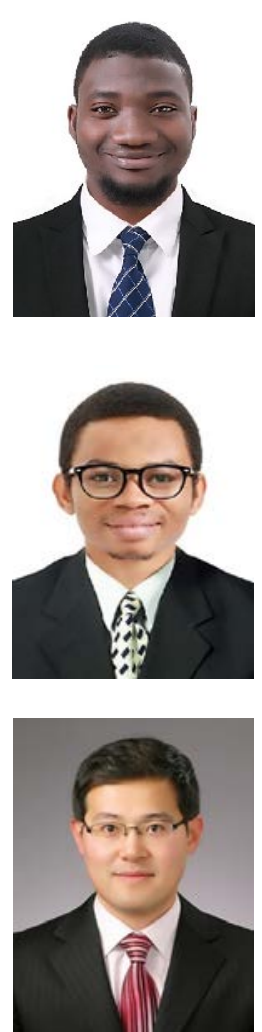

Alidu Abubakari received the B.S. degree in telecommunication engineering from the Kwame Nkrumah University of Science and Technology, Kumasi, Ghana, in 2013, and the M.S. degree in electronic engineering from Hanbat National University, Daejeon, South Korea, in 2016 where he is pursuing a Ph.D. degree in electronic engineering. He is currently a Researcher in substation automation and smart grid communication at Korea Electric Power Company, Daejeon, South Korea. His research interests include distributed massive MIMO networks (3-D spatial channel modeling, beamforming, and SDMA), cloud radio access networks, small cells, smart grid and heterogeneous networks.

Sabogu-Sumah Raymond was born in Ghana. He received the B.Sc. degree in telecommunications engineering (First Class Hons) from the Kwame Nkrumah University of Science and Technology, Kumasi, Ghana, in 2013. He is currently pursuing the master's degree with Hanbat National University, Daejeon, South Korea. He was with Huawei Technologies and National Communications Authority both in Ghana as a Network Optimization and Telecommunications Engineer, respectively. His research interests include spectrum sharing, coexistence between wireless services, millimeter wave communications, and cognitive radio networks.

Han-Shin Jo received the B.S., M.S., and Ph.D. degrees in electrical and electronics engineering from Yonsei University, Seoul, South Korea, in 2001, 2004, and 2009, respectively. He is currently an ssociate Professor with the Department of Electronics and Control Engineering, Hanbat National University, Daejeon, South Korea. He was a Post-Doctoral Research Fellow with the Wireless Network and Communications Group, Department of Electrical and Computer Engineering, University of Texas at Austin, from 2009 to 2011. He developed a long-term evolution base station in Samsung Electronics from 2011 to 2012. His current research interests are in all the aspects of MIMO (channel modeling, precoding and scheduling with limited feedback, beamforming, SDMA, and massive MIMO) and applications of stochastic geometry and optimization theory to wireless cellular and ad hoc networks. He received the Samsung Electronics Graduate Fellowship from 2006 to 2008, the Korea Research Foundation BrainKorea21 Graduate Fellowship from 2006 to 2007, the Korea Research Foundation Post-Doctoral Fellowship in 2009, and the 2011 ETRI Journal Best Paper Award. 